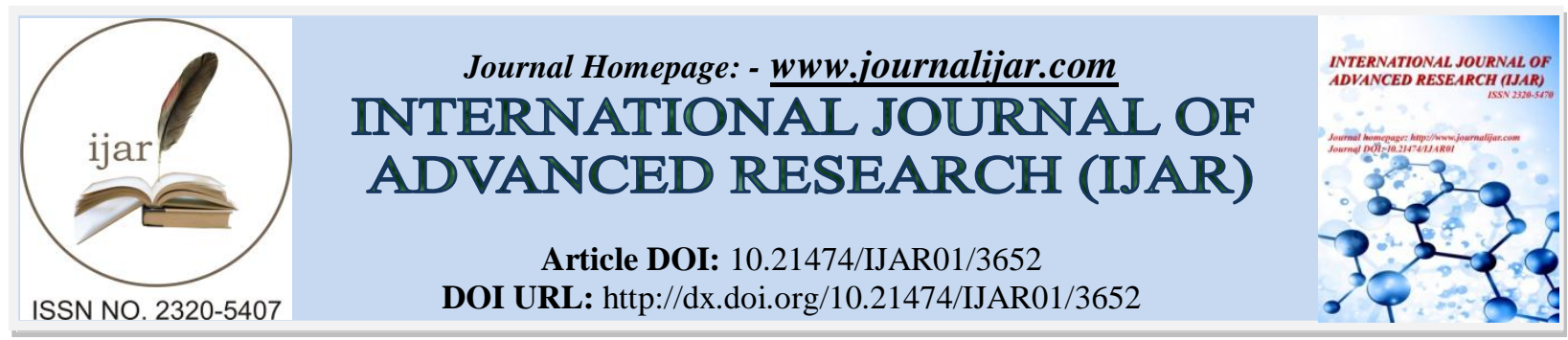

RESEARCH ARTICLE

\title{
THERMO ACOUSTIC STUDY OF BINARY LIQUID MIXTURES OF DIMETHYL SULFOXIDE AND TRIETHYLAMINE BY ULTRASONIC INTERFEROMETRIC TECHNIQUES.
}

\section{Shweta Rode, V. D. Bhandakkar ${ }^{1}$ and O. P. Chimankar ${ }^{2}$.}

1. Department of Electronics, Anand Niketan College, Warora, India 442907.

2. Department of Physics, RTM, Nagpur (MS), India (40001).

\section{Manuscript Info}

Manuscript History

Received: 10 January 2017

Final Accepted: 01 February 2017

Published: March 2017

Key words:-

Ultrasonic velocity, Acoustical properties, Molecular interactions.

\begin{abstract}
The ultrasonic velocity, density and viscosity have been measured in the binary systems of dimethyl sulfoxide (DMSO) with triethylamine (TEA). From the experimental data, various acoustical parameters such as adiabatic compressibility (b), intermolecular free length $\left(\mathrm{L}_{\mathrm{f}}\right)$, free volume $\left(\mathrm{V}_{\mathrm{f}}\right)$, internal pressure $\left(\pi_{\mathrm{i}}\right)$, Relaxation time $(\tau)$, Acoustic impedance $(Z)$, Gibb's free energy $(\Delta \mathrm{G})$ and Vander Waal's constant (b) were calculated. The results are interpreted in terms of molecular interaction between the components of the mixtures.
\end{abstract}

Copy Right, IJAR, 2017. All rights reserved.

\section{Introduction:-}

In recent years, ultrasonic technique has become a powerful tool for studying the molecular behavior of liquid mixtures [1-3]. The measurements of ultrasonic velocity have been adequately employed in understanding the molecular interactions in liquid mixtures. Molecular interaction studies can be carried out by both spectroscopic [45] and non-spectroscopic [6-7] techniques. A large number of studies have been made on the molecular interaction in liquid systems by various physical methods like, Raman Effect, Nuclear Magnetic Resonance, Ultra Violet and ultrasonic method [8-10]. However, ultrasonic velocity and viscosity measurements have been widely used in the field of interactions and structural aspect evaluation studies. In recent years ultrasonic technique has become powerful tool in providing information regarding the molecular behavior of liquids and solids, owing to its ability of characterizing Physico-chemical behavior of the medium. The ultrasonic velocity data for binary and ternary liquid mixtures have been used for by many researchers [11-14].

In order to have a clear understanding of the intermolecular interactions between the component molecules, the authors have performed thorough study on the liquid mixtures using ultrasonic velocity data. The present work deals with the ultrasonic velocity and computation of related parameters in binary system of dimethyl sulfoxide + triethylamine at $298.15 \mathrm{~K}, 303.15 \mathrm{k}$ and $308.15 \mathrm{~K}$ at frequency $7 \mathrm{MHz}$. For preparing various concentration mixtures, mole fractions of component liquids were varied from 0.0 to 1.0. Dimethyl sulfoxide, as a polar solvent, is certainly too some extent associated by dipole-dipole interactions, and is of particular interest because of the absence of any significant structural effects due to the lack of hydrogen bonds; therefore, it may work as an aprotic, protophilic solvent with a large dipole moment and high dielectric constant. 


\section{Results and Discussions:-}
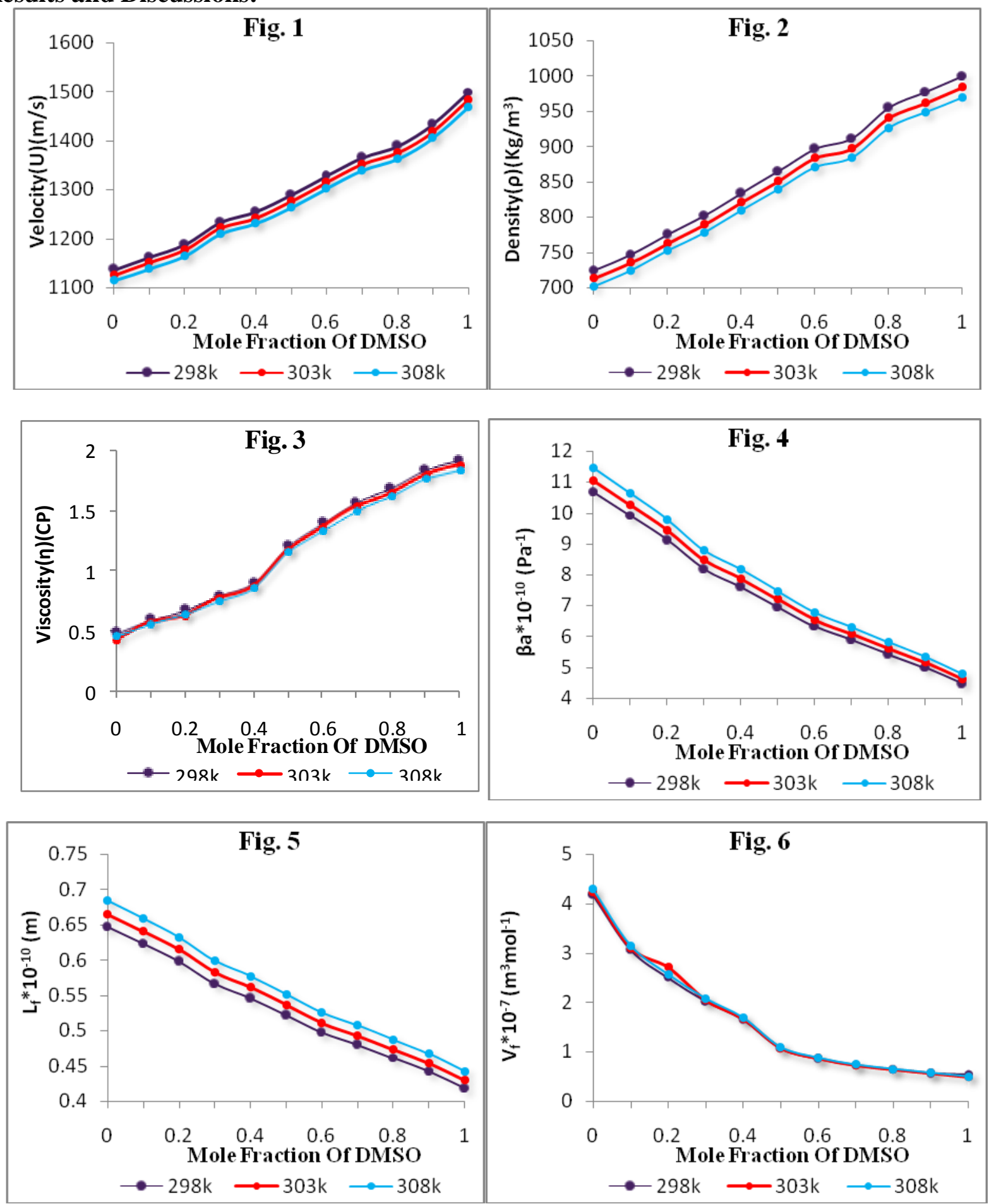

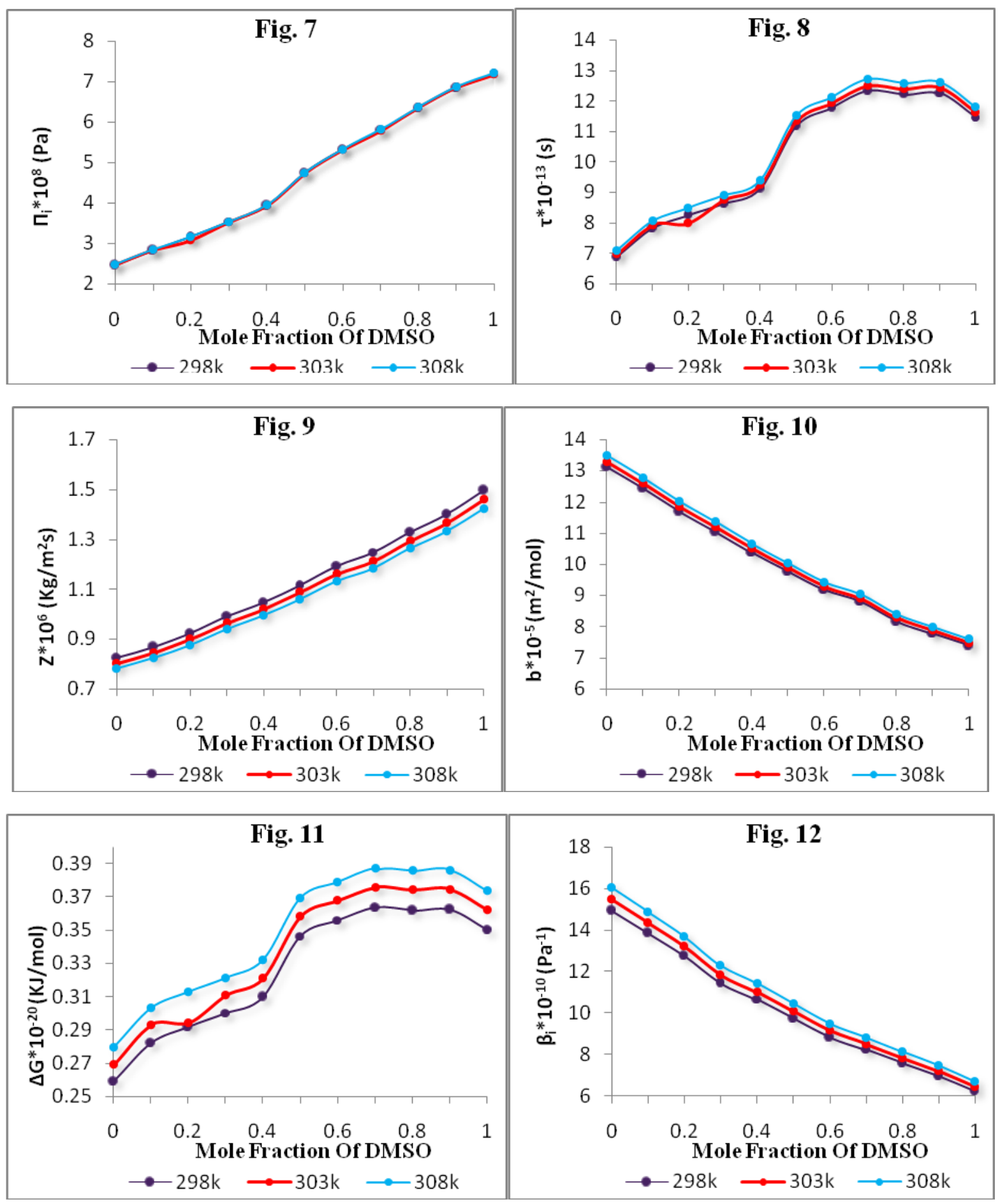

The variation of density, viscosity and ultrasonic velocity Vs mole fraction for the binary system dimethyl sulfoxide +triethylamine at $298.15 \mathrm{~K}, 303.15 \mathrm{~K}$ and $308.15 \mathrm{~K}$ are shown in fig.-1, 2 and 3 respectively. While other thermodynamics parameters such as adiabatic compressibility $\left(\beta_{\mathrm{a}}\right)$, free length $\left(\mathrm{L}_{\mathrm{f}}\right)$ free volume $\left(\mathrm{V}_{\mathrm{f}}\right)$ internal pressure $\left(\pi_{\mathrm{i}}\right)$, relaxation time $(\tau)$, and acoustic impedance (Z), Gibb's free energy $(\Delta \mathrm{G})$, Vander Waal's constant (b), Isothermal compressibility $\left(\beta_{\mathrm{i}}\right)$ at temperature $303 \mathrm{~K}$ are shown in fig.-4,5,6,7,8 9,10,11 and 12 respectively. 
Fig. 1 shows the graph of ultrasonic velocity versus concentration. It is observed that ultrasonic velocity increase with increase in the concentration of DMSO in TEA, indicating association in constituent's molecules. The association may involve due to hydrogen bonding or dipole-dipole interaction between DMSO in TEA.

Fig.2 shows the graph of density versus concentration. It is observed that density increases with increase in concentration of DMSO in TEA. Increase in density decrease in volume, indicating association in component molecules. The density of binary liquid mixtures may be increase due to structural reorganization indicating the closed packed structure of the molecules cluster. This makes the liquid medium less compressive. Increasing temperature of the mixture decreases its density $(\rho)$. The decrease in density $(\rho)$ with temperature indicates decrease in cohesive force. Thus increase of temperature favors increase of kinetic energy and volume expansion and hence decrease of density.

Fig. 3 consists of viscosity $(\eta)$ versus concentration. It is observed that viscosity increases with increase in the concentration of DMSO in TEA, indicating strong molecular interaction. The viscosity gives the strength of molecular interaction between interacting molecules. Increase in temperature of the mixture, increasing disorders of the medium and hence entropy increases. As entropy increases, viscosity of binary mixture decreases.

Free Length, Increase in concentration of DMSO thus results in decrease in adiabatic compressibility $\left(\beta_{\mathrm{a}}\right)$ and free length $\left(\mathrm{L}_{\mathrm{f}}\right)$ as shown in Fig. 3 and Fig 4. The regular fall in free length with increase in concentration of DMSO causes a rise in sound velocity [16]. This trend is an indication of clustering together of the molecules as the associative effect of the polar group dominates over the other type of interaction [17]. Velocity shows a reverse trend as temperature is increased. This happens as the spacing between the molecules increases leading to a less ordered structure.

The adiabatic compressibility $\left(\beta_{\mathrm{a}}\right)$ and Isothermal compressibility $\left(\beta_{\mathrm{i}}\right)$ exhibit similar trend. From Fig.4 and 12, it is observed that adiabatic compressibility $\left(\beta_{\mathrm{a}}\right)$ and isothermal compressibility $\left(\beta_{\mathrm{i}}\right)$ decreases with increase in molar concentration of DMSO in TEA indicating strong intermolecular interaction in the component molecules in binary mixtures shows associating tendency of the component molecules.

Fig. 6, it is observed that free volume decreases $\left(V_{f}\right)$ with increase in mole concentration of DMSO in TEA, indicating association in the molecules of the component liquids. It was observed that the variation in the free volume values show exactly the reverse trend as that of internal pressure as shown in Fig. 7, gradually increases indicating weak molecular interaction amongst DMSO in TEA [18]. In the present paper, it is observed that free volume decreases and internal pressure increases.

Increase in internal pressure (Пi) may be due to strengthening of cohesive force. Since the interaction in our case is weak, ' $\Pi_{\mathrm{i}}$ ' increases slowly with concentration of DMSO, but decreases rapidly with increase of temperature.

Relaxation time $(\tau)$ increases with increase in mole fraction of DMSO as presented in Fig. 8, but increases as temperature increases. The former indicates weak molecular interaction between the components of the mixture, and the latter is true because of instantaneous conversion of excitation energy to translational energy. . The relaxation time which is in the order of $10^{-13} \mathrm{sec}$ is due to structural relaxation process [19] and in such a situation, it is suggested that, the molecules get rearranged due to co-operative process [20]. Relaxation time is the time taken for the excitation energy to appear as translational energy and it depends on temperature and impurities.

Acoustic impedance $(\mathrm{Z})$ is the ratio of the effective sound pressure at a point to the effective particular velocity at that point. The pressure is measured by the totality of the force of dispersion, repulsion, ionic and dipolar. In our present investigation, From Fig. 9, acoustic impedance increases with increase in concentration of DMSO, showing weak molecular interaction. Since $\mathrm{Z}=\mathrm{U} \rho$, as temperature increases, $\mathrm{Z}$ decreases. Acoustic impedance is also used for assessing the absorption of sound in the medium.

Fig. 10 represents the Vander Waal's Constant (b). Vander Waal's constant (b) is also known as the co-volume in in Vander Waal's equation and it varies in similar way to that of the available volume. The co-volume decrease with increasing concentration of DMSO in TEA indicating strong interaction between the component molecules. Also the linear variation of these acoustical properties indicating absence of complex formation in the mixture. These 
parameters help for the study of range of Repulsive [21] and attractive forces in the molecules of the components. The change in Vander Waal's Constant (b) would be due to a change in intermolecular geometry (micro geometry).

Gibb's free energy measures mobility of the medium. Higher the mobility of the medium, higher will be the entropy; lower will be the free energy. Fig. 11represents the variation of Gibb's free energy with concentration. It is observed that Gibb's free energy increase with increase in concentration DMSO in TEA, indicating decrease in mobility of the mixture hence disorder decreases. This leads to the low entropy and hence salvation increase. This is because of the fact that salvation is directly proportional to size of the molecule.

\section{Conclusions:-}

Density, viscosity and ultrasonic velocity increases with increase in mole fraction of dimethyl sulfoxide in triethylamine. This is due to the dipole - dipole interaction between dimethyl sulfoxide and triethylamine components. The decrease in adiabatic compressibility and free length for the system- dimethyl sulfoxide in triethylamine is due to strong association. The dipole-dipole interactions between dimethyl sulfoxide and triethylamine are found to be responsible for association in the binary liquid mixture.

\section{References:-}

1. P S Nikam, B S Jagdale, A B Sawant and Mehdi Hasan, Indian J Pure Appl Phys., 39,(2001), 433.

2. Shipra Baluja and Swati Oza, Fluid Phase Equilib. 178, (2001), 233.

3. A Ali, A K Nain and M Kamil, Thermochim. Acta, 274, (1996), 209.

4. C V Suryanarayana, J Acoust Soc Ind., 13,(1983), 11.

5. Fletcher A, J Phys Chem., 73,(1969), 2217.

6. G C Pimental and A L Maclellan, "The Hydrogen Bond, (WH Freeman and Co, San Fransisco) (1960).

7. M E Hobbs and W W Bates, J Am Chem Soc., 74, (1952), 746.

8. O P Chimankar, R Shriwas and V A Tabhane, J. Chem Pharm Res, 3(3),(2011),587-596.

9. A N Kannappan \& V Rajendran, Indian J. Pure \&Applied Physics, 30,(1992), 176.

10. V D Bhandakkar et al, J. Pure appl. \& Ind. Phys. 3(2),(2013), 80-86.

11. O P Chimankar, K G Rewatkar \& V A Tabhane, Indian J. of Phys., 75 B (2), (2001),141-145.

12. V D Bhandakkar, O P Chimankar \& N R Pawar, J. of Chemical \& Pharmaceutical Research, 4,(2010), 873877.

13. G R Bedare, V D Bhandakkar and B M Suryavanshi, IJAPM, 2(3),( 2012),197-200.

14. O P Chimankar, V A Tabhane, \& G K Beghel, J. Acoust Soc Of India, 34(4), (2007),126.

15. J Vogal, Practical organic chemistry, 4th edn. Longman, London. (1978).

16. H Eyring\& J F Kincaud, J. Chem Phys, , DOI: 10.1063/1.1750134, 6, (1938), 620-629.

17. B Hemalatha, Ph.D thesis, Annmalai University, (2004).

18. Dhana Lakshmi and Sekhars, Ind J. Pure \& Appl Ultrasons, 21, 3, (1999), 97.

19. L E Kinser and A R Fray, Fundamentals of Acoustics. New Delhi Eiley Eastern,(1989).

20. Ali Hyder S and A K Nain 200. J. Phys. 74(B1), 63-67.

21. G K Johri \& R L Mishra, Ind. J Pure \& Appl Phys, 22,(1984), 677. 